\title{
Cooperation and acting for the greater good during the COVID-19 pandemic
}

Valerio Capraro ${ }^{1}$, Paulo S. Boggio ${ }^{2}$, Robert Böhm ${ }^{3,4,5}$, Matjaž Perc ${ }^{6,7,8,9}$, Hallgeir Sjåstad $^{10}$

\author{
${ }^{1}$ Economics Department, Middlesex University London \\ ${ }^{2}$ Mackenzie Presbyterian University, São Paulo, Brazil \\ ${ }^{3}$ Department of Psychology, ${ }^{4}$ Department of Economics, ${ }^{5}$ Copenhagen Center for Social \\ Data Science (SODAS), University of Copenhagen, Denmark \\ ${ }^{6}$ Faculty of Natural Sciences and Mathematics, University of Maribor, Koroška cesta 160, \\ 2000 Maribor, Slovenia \\ ${ }^{7}$ Complexity Science Hub Vienna, Josefstädterstraße 39, 1080 Vienna, Austria \\ ${ }^{8}$ Department of Medical Research, China Medical University Hospital, China Medical \\ University, Taichung 404332, Taiwan \\ ${ }^{9}$ Alma Mater Europaea, Slovenska ulica 17, 2000 Maribor, Slovenia \\ ${ }^{10}$ Department of Strategy and Management, Norwegian School of Economics, Bergen, \\ Norway
}

Capraro, V., Boggio, P. S., Böhm, R., Perc, M., \& Sjåstad, H. (forthcoming). Cooperation and acting for the greater good during the COVID-19 pandemic. In M. K. Miller (Ed.) The

Social Science of the COVID-19 Pandemic: A Call to Action for Researchers. Oxford: Oxford University Press.

\begin{abstract}
Cooperation is essential to overcome global pandemics and other health crises, as many preventative measures require people to bear a personal cost to benefit other people or society as a whole. Wearing a face mask, for example, protects other people more than it protects oneself. In this essay, we discuss several mechanisms that are known to promote cooperation in economic games and real-world scenarios, with a special focus on message-based interventions that might be helpful to promote social distancing, mask wearing, physical hygiene, vaccine uptake, and information seeking. Lessons learned and future research ideas are offered.
\end{abstract}

\section{Keywords}

COVID-19, mask wearing, social distancing, vaccine hesitation, information seeking, physical hygiene, cooperation, policy interventions, message-based interventions. 


\section{Introduction}

As we write this, the novel Coronavirus has led to the greatest pandemic since the "Spanish flu" a century ago (1918-1920). Termed COVID-19, the new pandemic has so far infected more than 170 million people worldwide, with a preliminary death rate exceeding 3 million. At all stages of the collective effort to defeat the virus, cooperation is essential. In this book chapter, we therefore outline some key characteristics of successful cooperation, before we suggest how such principles can inform effective interventions during the current pandemic and future health crises requiring large-scale behavior change.

\section{COVID-19: A cooperative super-challenge}

In response to the COVID-19 pandemic and future pandemics, cooperation is essential. Identifying the initial threat; sharing relevant information; implementing policy interventions to initiate large-scale behavior change and "flatten the infection curve" (e.g., lockdowns of society in critical periods, mask wearing, improved physical hygiene and social distancing); developing evidence-based vaccines that are both safe and effective; distributing the vaccines as soon as they are ready; and finally, making sure that the majority of the public actually decide to take the vaccine. When facing a global pandemic like COVID-19 none of these stages can succeed without effective cooperation, since each stage requires a combination of personal costs and social coordination to achieve a greater goal. This makes psychology, behavioral economics, and social science a relevant starting point for how to approach the problem (Van Bavel et al., 2020a).

Just like many other domains in social life, the COVID-19 pandemic represents a "public goods dilemma": When most people cooperate most of the time, the larger group can succeed at defeating the virus. If a sufficiently large minority refuses to cooperate, however, the problem may persist or get much worse. In our understanding, 'cooperation' is characterized by the willingness to incur personal costs to help someone else, in which mutual cooperation generates a greater sum of resources and better outcomes than each party could achieve alone (Henrich \& Henrich, 2007; Nowak, 2006).

The ideal form of cooperation is often referred to as positive-sum interaction, as the total benefits outweigh the costs in the long run (Morgenstern \& Von Neumann, 1953; Trivers, 1971; Axelrod \& Hamilton, 1981). When people perceive a given situation this way, they are likely to engage in helping behavior (Ent et al., 2020). However, as cooperation 
involves an immediate cost and the greater reward is usually delayed or uncertain, there is always a risk that free-riding may overturn the initial willingness to share resources and help each other (Wang et al. 2016; Chinazzi et al. 2020; Aleta et al. 2020; Van Bavel et al. 2020a). For instance, mask wearing in public spaces has been found to be an effective intervention to reduce infection spread (Mitze, Kosfeld, Rode, \& Wälde, 2020), but the greatest benefit appears to manifest for the people surrounding the mask-wearing person. When it comes to social distancing and adherence to public lockdowns, young people have probably made the largest sacrifice by implementing the greatest changes in their daily lives (which normally is very social at that age), whereas the elderly and people with pre-existing health conditions have received the greatest benefits from these interventions as they are at highest mortality risk. Another important example regards vaccine uptake. Clearly, vaccination during a pandemic should be quite attractive to people because it allows getting back to "normal" even for those with low risk of severe infection, who are nevertheless affected by the behavioral regulations imposed on them. However, this happens only if the majority of other people get vaccinated as well. Moreover, compared to other vaccines against well-known diseases, newly developed vaccines could be perceived as less trustworthy and misinformation around COVID-19 may further undermine vaccination intentions (Loomba et al., 2021; Freeman et al., 2021; Kaplan \& Milstein, 2021; Dodd et al., 2020; Gozum, 2021). This combination of personal costs and social benefits suggests that vaccination also has a prosocial value (Betsch et al., 2015; Korn et al. 2020). In line with this view, Wells et al. (2020) found that Polio vaccination in Israel was mainly attributable to prosocial motivation. Finally, practices of physical hygiene (such as handwashing and sneezing on one's sleeves) and information seeking have also been shown to correlate with prosocial behavior during the COVID-19 pandemic (Boggio et al. 2021; Campos-Mercade et al. 2021).

In light of these reflections on the cooperative nature of pandemic responses, we will take a closer look at the underlying logic of successful cooperation based on relevant research from economic games and real-life scenarios. First, we will outline some key principles of cooperative behavior in general. Then we will review how these principles can be used to design message-based interventions to promote pandemic response. 


\section{The fundamental mechanisms of cooperative behavior}

\section{Kin selection}

Humans (and other animals) are more likely to incur personal costs to help genetic relatives than non-genetic cooperation partners. Due to the principle of "inclusive fitness", genetic self-interest motivates people to not only secure their own survival, but also the survival and well-being of relatives sharing similar genes (Hamilton, 1964). In consequence, people are more likely to cooperate when it is clear that it will benefit their loved ones. Even simple cues that suggest genetic kinship have been found to increase cooperation in public goods games (Krupp, Debruine, \& Barclay, 2008).

\section{Direct reciprocity}

Direct reciprocity is a form of "tit-for-tat" strategy in repeated interactions between the same individuals over time. This is a widely observed pattern: People are strongly motivated to return favors and previous helping behavior, and conversely, to defect from future cooperation with individuals who have violated their trust in the past (Gouldner, 1960; Cialdini, 2009; Nowak, 2006). So-called "end-game effects" refers to the common drop in cooperation towards the end of repeated interactions, meaning that, when there is no possibility for future reciprocity, people tend to cooperate less. On the other hand, the "shadow of the future" typically increases cooperation (Dal Bó, 2005; Camera \& Casari, 2009; Van Lange, Klapwijk, \& Van Munster, 2011), and even just thinking about the future makes people more willing to share resources with others (Sjåstad, 2019).

\section{Indirect reciprocity}

Indirect reciprocity represents a form of social learning, in which third parties may punish or reward cooperators or defectors through reputational information (Nowak \& Sigmund, 1998; Milinski et al. 2001; Gächter \& Falk, 2002; Fehr, 2004; Nowak \& Sigmund, 2005; Nax et al. 2015). Recent research has confirmed that reputation is a powerful motive in social decision-making, as people often assume they are being observed by potential cooperation partners even when they make anonymous choices (Jordan \& 
Rand, 2020). Thinking about the future can amplify this reputational concern further (Vonasch \& Sjåstad, 2021). One implication is that, whenever people's behavior is observable and identifiable to others, cooperative behavior tends to increase. Conversely, when people are less visible or perhaps not identifiable at all, adaptive group functioning tends to suffer and decline (Baumeister, Ainsworth, \& Vohs, 2016).

\section{Cost and benefit of cooperation}

The definition of cooperation itself suggests that cooperation rates might depend negatively on the cost of cooperation and positively on the benefit created. Experiments on the one-shot prisoner's dilemma have confirmed that people are more likely to cooperate when the cost of cooperation decreases (Engel \& Zhurakhovska, 2014) and when the benefit of cooperation increases (Capraro, Jordan, \& Rand, 2014). This suggests that also making salient the social benefits (or downplaying the costs) of cooperation may increase cooperative behavior. For example, Dal Bó \& Dal Bó (2014) found that having people read an "utilitarian message" that makes salient that cooperating maximizes the group payoff, increases cooperation in the iterated prisoner's dilemma.

\section{Norms}

Cooperative behavior is also driven by a desire to follow a norm (Biziou-van-Pol et al. 2015; Kimbrough \& Vostroknutov, 2016; Capraro \& Rand, 2018). See Capraro and Perc (2021) for a review. This suggests that making salient the normative value of an action can increase cooperative behavior. Accordingly, making people read the Golden Rule increases cooperative behavior in the iterated prisoner's dilemma (Dal Bó \& Dal Bó, 2014) and asking people to report what they think is the morally right thing to do, or what they think others think is the morally right thing, increases cooperation in the one-shot prisoner's dilemma (Capraro et al. 2019).

\section{Social heuristics}

The Social Heuristics Hypothesis states that people internalise heuristics that are successful in everyday interactions and use them in situations where they do not possess 
enough cognitive resources to compute their payoff maximizing strategy. Since most of our real-life interactions are repeated, this framework predicts that people tend to internalize cooperative heuristics (Rand et al. 2014). Accordingly, promoting intuition tends to increase cooperative behavior in one-shot economic games played in the lab (Rand, 2016), especially among inexperienced subjects (Rand et al. 2014) and those who trust those around them (Rand \& Kraft-Todd, 2014). Although this finding has also been criticized (Kvarven et al. 2019), scholars agree that nudging people to rely on their emotion increases cooperative behavior (Levine et al. 2018; Kvarven, 2019; Rand, 2016). See Capraro (2019) for a review.

\section{Leadership}

The role of leaders is fundamental to promote collective changes, especially when they are costly for the individual. When there is ambiguity about what is the right thing to do in a given situation, as it may happen during a public health crisis, people may look out at leaders to find out how to behave. The experimental literature using economic games has demonstrated that good examples by leaders can improve cooperation (Haigner \& Wakolbinger, 2010), whereas poor examples can decrease cooperation (Moxnes \& vander Heijden, 2003). Moreover, "leading by example" has a greater positive effect than leading by words on cooperation in a public goods game (Dannenberg, 2015). That said, trust in leaders is a key moderator. For example, during the Ebola outbreak, trust in institutions was associated with the decisions to abide by social distancing mandates in Liberia (Blair, Morse, \& Tsai, 2017) and vaccination mandates in Congo (Vinck et al. 2019).

\section{Group selection}

The human population is obviously divided in groups (e.g., nations). This group structure may lead to the evolution of cooperation as follows. Assume that a group made by cooperators grows faster than a group made by defectors. If there are constraints on the total population size, then smaller, defective, groups may become extinct, as cooperative groups grow larger. This logic may lead to the evolution of cooperation, despite the centrifugal within-group forces that drive individuals towards defection (Nowak, 2006). Group 
selection is psychologically based on group identity. This suggests that making group identity salient may increase cooperative behavior (Dawes, Van De Kragt, \& Orbell, 1988).

\section{Network reciprocity}

Modern human societies are built upon social networks (Christakis \& Fowler, 2009). Although these networks change over time (Perc \& Szolnoki, 2009; Holme \& Saramäki, 2012), they nevertheless introduce a limited interaction range to our existence that significantly shapes our cooperative behavior. In fact, an important mechanism for cooperation is network reciprocity (Nowak \& May, 1992), which stands for the fact that a limited interaction range facilitates the formation of compact clusters of cooperators that are in this way protected against invading defectors. This basic mechanism can be enhanced further if the degree distribution of the social network is strongly heterogeneous (Santos \& Pacheco, 2005; Gómez-Gardeñes et al. 2007), if there is a set or community structure (Tarnita et al. 2009; Fotouhi et al. 2019), or if the evolution unfolds on two or more network layers that mutually support cooperative clusters (Wang, Szolnoki, \& Perc, 2012; Gómez-Gardeñes et al. 2012; Battiston et al. 2017; Fu \& Chen, 2017; Fotouhi et al. 2018). This adds to a long line of mechanisms for cooperation on networks, ranging from simple coevolutionary rules that may affect the structure of the interaction network, the teaching activity of individuals, their reputation, mobility, or age (Santos, Pacheco, \& Lenaerts, 2006), to various forms of heterogeneity, that arises as a consequence of these rules or is inherently present in a population (Santos, Santos, \& Pacheco, 2008; Perc \& Szolnoki, 2008; Santos et al., 2012; Amaral et al., 2016). See Perc et al. (2017) for a review.

\section{Higher-order network reciprocity}

Despite the wealth of important insights concerning cooperation on networks, an important unsolved problem remained accounting for cooperation in groups, such as for example in the public goods game (Archetti \& Scheuring, 2012; Perc et al., 2013). The simplest remedy was to consider members of a group to be all the players that are pairwise connected to a central player (Santos, Santos, \& Pacheco, 2008; Szolnoki, Perc, \& Szabó, 2009). However, since the other players are further connected in a pairwise manner, one would also need to consider all the groups in which the central player is a member but is not central. Evidently, classical networks do not provide a unique procedure for defining a 
group. Moreover, members of the same group are commonly not all directly connected with one another, which prevents strategy changes among them. These facts used to posit a lack of common theoretical foundation for studying the evolution of cooperation in networked groups. Without knowing who is connected to whom in a group, it was also impossible to implement fundamental mechanisms that promote cooperation.

Recently, a solution came in the form of higher-order networks, where, unlike in classical networks (Latora, Nicosia, \& Russo, 2017), a link can connect more than just two individuals (Battiston et al. 2020). Thus, higher-order networks naturally account for structured group interactions, wherein a group is made up of all players that are connected by a hyperlink (Berge, 1984). It has been shown that the public goods game on a uniform hypergraph corresponds to the replicator dynamics in the well-mixed limit, thus providing a formal theoretical foundation to study cooperation in networked groups (Alvarez-Rodriguez et al. 2021). Moreover, the presence of hubs and the coexistence of interactions in groups of different sizes can critically boost cooperation (Perc et al. 2017).

\section{How to promote cooperation during a pandemic}

Now we focus more specifically on what type of interventions may support a cooperative response to the COVID-19 pandemic. Specifically, our focus will be on message-based interventions intended to promote social distancing, physical hygiene, mask wearing, vaccine uptake, and information seeking. We consider these behaviors because they are key to fight the COVID-19 pandemic and they have all been shown to correlate with prosocial behavior and intentions (Boggio et al. 2021; Campos-Mercade et al. 2021; Coroiu et al. 2020; Jordan, Yoeli, \& Rand, 2020; Lu, Jin, \& English, 2021; Nivett et al. 2020). We focus on message-based interventions because they represent a powerful means to reach the population and induce collective changes, because they can be displayed in the street through posters and screens, or reach people inside their homes through social media, television, and radio.

\section{Social distancing}

Five studies found that prosocial messages are more effective than proself messages at increasing intentions to practise social distancing: Deslatte (2020) found that public-health frames increase intentions to avoid unnecessary travels; Pfattheicher et al. (2020) reported 
that inducing empathy increases intentions to practise social distancing; Lunn et al. (2020) found that prosocial messages highlighting that violating social distancing rules can lead to the infection of others increased intentions to practice social distancing; Heffner, Vives, \& FeldmanHall (2020) found that a prosocial message increased willingness to self-isolate; Cucchiarini et al. (2021) established that nudging the injunctive norm positively affects intentions to comply with physical isolation, especially among people with low-risk perception. On the other hand, two studies found that messages that highlight that the coronavirus is a threat to people's community do not increase intentions to practise social distancing, compared to the baseline or to proself messages (Capraro \& Barcelo, 2020; Jordan et al. 2020). A third study found that priming prosocial motivations, through messages highlighting that we can stop the spread only if we work together, did not affect intentions to practice social distancing (Favero \& Pedersen, 2020).

Some works tested messages highlighting consequences on close others, or kinship. Christner et al. (2020) found that moral judgments and empathy for loved ones are associated with intentions to practise social distancing, beyond self-oriented factors. However, Capraro and Barcelo (2020) found that a close prosocial message highlighting that the coronavirus is a "threat to your family" does not increase intentions to practise social distancing, compared to the baseline or a proself message.

Abu-Akel, Spitz, and West (2021) explored the role of different spokespersons on people's intentions to share a message calling for social distancing. Across six countries, they found that Dr. Anthony Fauci reached the highest level of resharing, followed by government spokespersons, and popular celebrities. This is in line with correlational work

finding that trust in experts is associated with intentions to comply with social distancing, more than trust in institutions (Ahluwalia, Edelen, Qureshi, \& Etchegaray, 2021; Jørgensen, Bor, \& Petersen, 2021).

\section{Physical hygiene}

Despite the aforementioned positive correlation between prosociality and physical hygiene (Boggio et al. 2021; Campos-Mercade et al. 2021; Jordan et al. 2020), prosocial messages have not been found to promote intentions of washing hands properly (Hacquin, Mercier, \& Chevallier, 2020). One potential explanation for this lack of effect might be saturation: Jordan et al. (2020) found that a prosocial message that highlights that the 
coronavirus is a "threat to your community" is more effective at increasing intentions to engage in preventative measures including physical hygiene, than a proself message highlighting that the coronavirus is a "threat to you", only in the early stage of the pandemics, but not in later ones.

As far as we know, there have been no studies exploring the effect of close prosocial messages. We believe this to be an important direction for future work. Since practises of physical hygiene tend to benefit those with whom we are in close contact, it is possible that making salient the benefit to close others, or kinship, are more effective at promoting this particular kind of behavior.

\section{Mask wearing}

Several prosocial messages have been found to increase intentions to wear a face mask. Capraro \& Barcelo (2020) found that telling people that the coronavirus is a "threat to your community" increases intentions to wear a face covering, compared to telling them that the coronavirus is a "threat to you". They also explored other messages based on kinship ("threat to your family") and on group identity ("threat to your country"), but they did not significantly increase intentions to wear a face mask. Van der Linden and Savoie (2020) found that a prosocial message, highlighting that those who do not wear a face mask can infect people with whom they come into contact, increased intentions to wear a face mask, compared to a proself message highlighting that those who do not wear a face mask could take the virus from those with whom they come into contact. Pfattheicher et al. (2020) found that inducing empathy, by having people read a text regarding a woman with a rare immune disease being affected by the coronavirus, increased intentions to wear a face mask.

There has also been one work testing the effect of messages manipulating intuitive and deliberative decision making. Capraro and Barcelo (2021) found that telling people to "rely on their reasoning" increased their intentions to wear a face covering, compared to telling them to "rely on their emotion", and also compared to the baseline.

\section{Vaccine uptake}

Some works showed that prosocial intentions matter and can be used to increase vaccine uptake during a pandemic. A theoretical framework, known as the 5C model, lists 
"collective responsibility", defined as a willingness to protect others and contribute to the elimination of infectious diseases, as one of the key determinants of the decision to vaccinate (Betsch et al. 2018). Specifically related to COVID-19, Jung and Albarracín (2021) found that concern for others is more likely to relate to COVID-19 vaccination intentions in areas with low (vs. high) social density, potentially due to a greater perceived prosocial benefit of one's vaccination on others. Pfattheicher, Petersen, and Böhm (in press) found that people with knowledge about and belief in herd immunity as well as empathy for those most vulnerable to the virus are more motivated to get vaccinated against COVID-19. In a second study, Pfattheicher and colleagues found that providing information about herd immunity and inducing empathy promoted vaccination intentions. Schwarzinger et al. (2021) found that vaccine hesitancy was lower when the benefits associated with herd immunity are made salient. However, there have also been studies finding no effect of prosocial information on intentions to get vaccinated (Freeman et al. 2021; Sprengholz, Betsch, \& Böhm, in press). In particular, Freeman et al. (2021) tested the effect of ten message-based interventions on intentions to get vaccinated, including a message that highlighted the collective benefits of vaccination and a message that highlighted the individual benefit. They found that information type had no effect among people who are already willing to get vaccinated, or doubtful. However, among people who are strongly hesitant, highlighting the individual benefit increased vaccination intentions more than highlighting the collective benefit of not getting ill and of not transmitting the virus. Yet, there is also experimental evidence that strong material individual benefits, in the form of monetary rewards for getting vaccinated or fines for not getting vaccinated in case of compulsory/mandatory vaccination, may cause psychological reactance, specifically among people with negative attitudes towards vaccination (Betsch \& Böhm, 2016; Sprengholz et al., in press).

\section{Seeking and understanding official information}

Despite the positive correlation between prosocial behavior in economic experiments and information seeking during the COVID-19 pandemic (Campos-Mercade et al. 2021), two experimental studies found that prosocial messages do not increase seeking and understanding official information beyond proself messages. Banker \& Park (2020) found that a prosocial frame ("protect your community") was actually less effective than a proself frame ("protect yourself") in eliciting clicks on a Facebook post containing official 
recommendations; whereas a close prosocial frame ("protect your loved ones") was equally effective as the proself frame. Bilancini et al. (2020) tested the effect of three norm-based posters. The first poster contained a message designed to nudge the personal norm, "do what you think is right"; the second one contained a message designed to nudge the descriptive norm, "do what you think others are doing"; the third one contained a message designed to nudge the injunctive norm, "do what you think others approve of". The authors found that none of them increased understanding of official governmental rules, as measured through comprehension questions, compared to the baseline.

\section{Suggested interventions, lessons learned and future research}

As in catastrophes or other epidemics, the new coronavirus pandemic reveals something fundamental of our species: the ability to cooperate and help others even at a cost to ourselves. Zaki (2020), when describing this human characteristic, used the term compassion catastrophe. We are living the pandemic, or in other words, we are participating in a tragic and deadly social and biological experiment. As in many naturalistic research, we can learn a lot by observing the behavior of people and groups in the face of this tragedy. Examples from around the world show people's ability to organize spontaneously to help those in greatest need - ranging from donations of food and medications, making masks to even phone calls to those who live alone. At the same time, clandestine parties are observed with people flocking without protection, demonstrations against vaccination, among other examples. Thus, a fundamental question is how to foster cooperative behaviors for the protection against and combat of the pandemic. In this chapter, we reviewed the literature, both general and then with a special focus on message-based interventions intended to promote cooperative response to COVID-19.

As can be seen, one of the most important aspects for promoting coping behaviors to COVID-19, present in both correlational and experimental studies, was prosociality. Consistently, individual profiles of greater prosociality, or experimental manipulations fostering participant's prosociality, were associated or resulted in greater support and adherence to several protective behaviors to COVID-19.

In specific and common to the studies, it was found greater support for the use of masks both for more prosocial individuals and after presenting prosocial messages in comparison to more proself messages. Such data is an important information for the promotion of public policies to combat pandemics, since the use of a mask is one of the main tools for 
protecting oneself and others, but which depends on its widespread use in the whole community to be effective. Laboratory studies as well as analysis of the effects of mandatory masking policies have shown significant declines in the spread of SARS-CoV2 (Brooks \& Butler, 2021). Thus, investment in campaigns promoting prosocial behaviors associated with the use of masks seems to be effective in changing the population's behavior and positively impacting the control of the pandemic.

But in addition to the positive effect on the use of masks, prosociality and empathy also promoted important changes to face the pandemic, namely: physical distancing and reduction of unnecessary travel. However, this has not been observed in some studies, signaling the need for further research evaluating the effect of prosocial messages on physical isolation. In this context, it has also been observed that messages sent by expert leaders are more effective than those sent by governmental officials and celebrities. This suggests that people trust experts more than they trust politicians or celebrities, a finding that is confirmed also by correlational evidence. This could be a useful suggestion for policymakers.

The effectiveness of prosocial messages is less conclusive when it comes to physical hygiene, vaccine uptake, and information seeking. General, distant, prosocial messages do not seem to increase practises of physical hygiene. Since physical hygiene benefits people with whom we are in close contact, it is possible that close prosocial messages, highlighting the benefit to kinship or close others, might be more effective. Future work could test this hypothesis. The evidence regarding the effect of prosocial messages on intentions to get vaccinated is mixed, with some studies showing a positive effect, while others finding a null effect. At the same time, some studies found that proself messages are more effective, at least among strongly hesitant people. This suggests the existence of important moderators. Given this mixed evidence, policymakers should consider and test the potential positive and negative behavioral consequences of message-based interventions for vaccination before implementing them at large. Finally, information seeking is a relatively unexplored territory. We believe this to be an important gap in the scientific literature, because the way citizens seek and understand official information is key to combat the pandemic, as it prevents the spread of misinformation or the access to unofficial, often contradictory, information. There is some evidence that close prosocial messages, norm-based messages, and proself messages have similar effects in promoting information seeking, thus further work should explore the effectiveness of different mechanisms. 
Most message-based interventions are built on a handful of mechanisms that support cooperation: kin selection, group selection, cost and benefits of cooperation. However, these are not the only mechanisms known to be associated with cooperative behavior. For example, several forms of reciprocity (direct, indirect, network, higher-order network) are known to promote cooperative behavior under certain circumstances. New studies could test such effects on behaviors necessary to face the pandemic; for example, evaluating aspects such as reciprocity and reputation in the use of a mask in contexts that manipulate experimental variables such as the level of relationship between people (family, friends, strangers) and the degree of cooperation of each participant (cooperatives and freeriders). Future research should attempt to integrate epidemic models with social dilemmas on higher-order networks to obtain even more realistic insights into what it takes to resolve the dilemma of epidemic control (Glaubitz \& Fu, 2020). A deep understanding of behavioral change in disease control and prevention, and in particular large-scale human cooperation, is urgently needed and will surely help to better inform pandemic response in the future.

The role of leadership is also relatively underexplored, as we found only one paper testing the effect of spokesperson. Studies that evaluate the types of leadership as well as the messages that each one brings to their respective populations can teach us important lessons for facing future pandemics. Similarly, just one work tested message-based interventions aimed at increasing group identity. Since Van Bavel et al (2020b) found that national identity is one of the main predictors of social distancing and physician hygiene, future work should explore the effect of message-based interventions grounded on national identity or other group identities.

In sum, we emerged from this pandemic with some lessons and some challenges. It is evident how our species is able to respond cooperatively to tragic situations such as the new coronavirus pandemic. But it is also evident that we need to know more about how to keep cooperatives cooperating and, very importantly, encourage cooperative behavior in freeriders.

\section{Acknowledgments}

Paulo S. Boggio was supported by: Coordenação de Aperfeiçoamento de Pessoal de Nível Superior - Brasil (CAPES - Programa Institucional de Internacionalização) grant 88887.310255/2018-00 (PSB) - National Council for Scientific and Technological Development grant 309905/2019-2 (PSB). 
Matjaž Perc was supported by the Slovenian Research Agency (Grant Nos. P1-0403 and $\mathrm{J} 1-2457)$.

\section{References}

Abu-Akel, A., Spitz, A., \& West, R. (2021). The effect of spokesperson attribution on public health message sharing during the COVID-19 pandemic. PLoS ONE, 16, e0245100.

Ahluwalia, S. C., Edelen, M. O., Qureshi, N., \& Etchegaray, J. M. (2021). Trust in experts, not trust in national leadership, leads to greater uptake of recommended actions during the COVID-19 pandemic. Risk, Hazards \& Crisis in Public Policy.

Aleta, A., Martin-Corral, D., y Piontti, A. P., Ajelli, M., Litvinova, M., Chinazzi, M., ... \& Moreno, Y. (2020). Modelling the impact of testing, contact tracing and household quarantine on second waves of COVID-19. Nature Human Behaviour, 4, 964-971.

Archetti, M., \& Scheuring, I. (2012). Game theory of public goods in one-shot social dilemmas without assortment. Journal of Theoretical Biology, 299, 9-20.

Alvarez-Rodriguez, U., Battiston, F., de Arruda, G. F., Moreno, Y., Perc, M., \& Latora, V. (2021). Evolutionary dynamics of higher-order interactions in social networks. Nature Human Behaviour.

Amaral, M. A., Wardil, L., Perc, M., \& da Silva, J. K. (2016). Evolutionary mixed games in structured populations: Cooperation and the benefits of heterogeneity. Physical Review E, 93, 042304.

Axelrod, R., \& Hamilton, W. D. (1981). The evolution of cooperation. Science, 211, 1390-1396.

Banker, S., \& Park, J. (2020). Evaluating prosocial COVID-19 messaging frames: Evidence from a field study on Facebook. Judgment and Decision Making, 15, 1037-1043.

Battiston, F., Cencetti, G., Iacopini, I., Latora, V., Lucas, M., Patania, A., ... \& Petri, G. (2020). Networks beyond pairwise interactions: structure and dynamics. Physics Reports, 874, 1-92.

Battiston, F., Perc, M., \& Latora, V. (2017). Determinants of public cooperation in multiplex networks. New Journal of Physics, 19, 073017.

Baumeister, R. F., Ainsworth, S. E., \& Vohs, K. D. (2016). Are groups more or less than the sum of their members? The moderating role of individual identification. Behavioral and Brain Sciences, 39, 1-56.

Berge, C. (1984). Hypergraphs: combinatorics of finite sets. Elsevier.

Betsch, C., \& Böhm, R. (2016). Detrimental effects of introducing partial compulsory vaccination: experimental evidence. The European Journal of Public Health, 26, 378-381.

Betsch, C., Schmid, P., Heinemeier, D., Korn, L., Holtmann, C., \& Böhm, R. (2018). Beyond confidence: Development of a measure assessing the 5C psychological antecedents of vaccination. PloS ONE, 13, e0208601.

Betsch, C., Böhm, R., \& Chapman, G. B. (2015). Using behavioral insights to increase vaccination policy effectiveness. Policy Insights from the Behavioral and Brain Sciences, 2, 61-73. 
Bilancini, E., Boncinelli, L., Capraro, V., Celadin, T., Di Paolo, R. (2020). The effect of norm-based messages on reading and understanding COVID-19 pandemic response governmental rules. Journal of Behavioral Economics for Policy, 4, Special Issue 1, 45-55.

Biziou-van-Pol, L., Haenen, J., Novaro, A., Occhipinti Liberman, A., \& Capraro, V. (2015). Does telling white lies signal pro-social preferences? Judgment and Decision Making, 10, 538-548.

Blair, R. A., Morse, B. S., \& Tsai, L. L. (2017). Public health and public trust: Survey evidence from the Ebola Virus Disease epidemic in Liberia. Social Science \& Medicine, 172, 89-97.

Boggio, P. S., et al. (in preparation). The pandemic is a time for moral actions: Individual's morality predicts support of collective action to fight the pandemic in an international sample.

Brooks, J. T., \& Butler, J. C. (2021). Effectiveness of mask wearing to control community spread of SARS-CoV-2. JAMA, 325, 998-999.

Camera, G., \& Casari, M. (2009). Cooperation among strangers under the shadow of the future. American Economic Review, 99, 979-1005.

Campos-Mercade, P., Meier, A. N., Schneider, F. H., \& Wengström, E. (2021). Prosociality predicts health behaviors during the COVID-19 pandemic. Journal of Public Economics, 195, 104367.

Capraro, V. (2019). The dual-process approach to human sociality: A review. Available at SSRN 3409146.

Capraro, V., \& Barcelo, H. (2020). The effect of messaging and gender on intentions to wear a face covering to slow down COVID-19 transmission. Journal of Behavioral Economics for Policy, 4, Special Issue 2, 45-55.

Capraro, V., \& Barcelo, H. (2021). Telling people to "rely on their reasoning" increases intentions to wear a face covering to slow down COVID-19 transmission. Applied Cognitive Psychology.

Capraro, V., Jagfeld, G., Klein, R., Mul, M., \& Van De Pol, I. (2019). Increasing altruistic and cooperative behaviour with simple moral nudges. Scientific Reports, 9, 11880.

Capraro, V., Jordan, J. J., \& Rand, D. G. (2014). Heuristics guide the implementation of social preferences in one-shot Prisoner's Dilemma experiments. Scientific Reports, 4 , 6790.

Capraro, V., \& Perc, M. (2021). Mathematical foundations of moral preferences. Journal of the Royal Society Interface, 18, 20200880.

Capraro, V., \& Rand, D. G. (2018). Do the right thing: Experimental evidence that preferences for moral behavior, rather than equity or efficiency per se, drive human prosociality. Judgment and Decision Making, 13, 99-111.

Cialdini, R. B. (2009). Influence: Science and practice (Vol. 4). Boston, MA: Pearson education.

Chinazzi, M., Davis, J. T., Ajelli, M., Gioannini, C., Litvinova, M., Merler, S., ... \& Vespignani, A. (2020). The effect of travel restrictions on the spread of the 2019 novel coronavirus (COVID-19) outbreak. Science, 368, 395-400. 
Christakis, N. A., \& Fowler, J. H. (2009). Connected: The surprising power of our social networks and how they shape our lives. Little Brown Spark, New York.

Christner, N., Sticker, R. M., Söldner, L., Mammen, M., \& Paulus, M. (2020). Prevention for oneself or others? Psychological and social factors that explain social distancing during the COVID-19 pandemic. Journal of Health Psychology.

Coroiu, A., Moran, C., Campbell, T., \& Geller, A. C. (2020). Barriers and facilitators of adherence to social distancing recommendations during COVID-19 among a large international sample of adults. PloS ONE, 15(10), e0239795.

Cucchiarini, V., Caravona, L., Macchi, L., Perlino, F. L., \& Viale, R. (2021). Behavioral Changes After the COVID-19 Lockdown in Italy. Frontiers in Psychology, 12, 617315.

Bó, P. D. (2005). Cooperation under the shadow of the future: experimental evidence from infinitely repeated games. American Economic Review, 95(5), 1591-1604.

Dal Bó, E., \& Dal Bó, P. (2014). "Do the right thing:" the effects of moral suasion on cooperation. Journal of Public Economics, 117, 28-38.

Dannenberg, A. (2015). Leading by example versus leading by words in voluntary contribution experiments. Social Choice and Welfare, 44, 71-85.

Dawes, R. M., Van De Kragt, A. J., \& Orbell, J. M. (1988). Not me or thee but we: The importance of group identity in eliciting cooperation in dilemma situations: Experimental manipulations. Acta Psychologica, 68, 83-97.

Deslatte, A. (2020). To shop or shelter? Issue framing effects and social-distancing preferences in the COVID-19 pandemic. Journal of Behavioral Public Administration, 3, 1-13.

Dodd, R. H., Pickles, K., Nickel, B., Cvejic, E., Ayre, J., Batcup, C., ... \& McCaffery, K. J. (2021). Concerns and motivations about COVID-19 vaccination. The Lancet. Infectious Diseases, 21, 161-163.

Engel, C., \& Zhurakhovska, L. (2014). Conditional cooperation with negative externalities-An experiment. Journal of Economic Behavior \& Organization, 108, 252-260.

Ent, M. R., Sjåstad, H., von Hippel, W., \& Baumeister, R. F. (2020). Helping behavior is non-zero-sum: Helper and recipient autobiographical accounts of help. Evolution and Human Behavior, 41, 210-217.

Favero, N., \& Pedersen, M. J. (2020). How to encourage "Togetherness by Keeping Apart" amid COVID-19? The ineffectiveness of prosocial and empathy appeals. Journal of Behavioral Public Administration, 3, 1-18.

Fehr, E. (2004). Don't lose your reputation. Nature, 432, 449-450.

Fotouhi, B., Momeni, N., Allen, B., \& Nowak, M. A. (2018). Conjoining uncooperative societies facilitates evolution of cooperation. Nature Human Behaviour, 2, 492-499.

Fotouhi, B., Momeni, N., Allen, B., \& Nowak, M. A. (2019). Evolution of cooperation on large networks with community structure. Journal of the Royal Society Interface, 16, 20180677.

Freeman, D., Loe, B. S., Yu, L. M., Freeman, J., Chadwick, A., Vaccari, C., ... \& Lambe, S. (2021). Effects of different types of written vaccination information on COVID-19 
vaccine hesitancy in the UK (OCEANS-III): a single-blind, parallel-group, randomised controlled trial. The Lancet Public Health.

Fu, F., \& Chen, X. (2017). Leveraging statistical physics to improve understanding of cooperation in multiplex networks. New Journal of Physics, 19, 071002.

Gächter, S., \& Falk, A. (2002). Reputation and reciprocity: Consequences for the labour relation. Scandinavian Journal of Economics, 104, 1-26.

Glaubitz, A., \& Fu, F. (2020). Oscillatory dynamics in the dilemma of social distancing. Proceedings of the Royal Society A, 476, 20200686.

Gómez-Gardeñes, J., Campillo, M., Floría, L. M., \& Moreno, Y. (2007). Dynamical organization of cooperation in complex networks. Physical Review Letters, 98, 108103.

Gómez-Gardenes, J., Reinares, I., Arenas, A., \& Floría, L. M. (2012). Evolution of cooperation in multiplex networks. Scientific Reports, 2, 620.

Gouldner, A. W. (1960). The norm of reciprocity: A preliminary statement. American Sociological Review, 161-178.

Gozum, I. E. A. (2021). Common good and public service as vital components for government officials in promoting COVID-19 vaccination. Journal of Public Health.

Hacquin, A. S., Mercier, H., \& Chevallier, C. (2020). Improving preventive health behaviors in the COVID-19 crisis: a messaging intervention in a large nationally representative sample. https://psyarxiv.com/nyvmg/

Haigner, S. D., \& Wakolbinger, F. (2010). To lead or not to lead: Endogenous sequencing in public goods games. Economics Letters, 108, 93-95.

Hamilton, W. D. (1964). The genetical evolution of social behaviour. II. Journal of Theoretical Biology, 7, 17-52.

Heffner, J., Vives, M. L., \& FeldmanHall, O. (2020). Emotional responses to prosocial messages increase willingness to self-isolate during the COVID-19 pandemic. Personality and Individual Differences, 170, 110420.

Henrich, N., \& Henrich, J. P. (2007). Why humans cooperate: A cultural and evolutionary explanation. Oxford University Press.

Holme, P., \& Saramäki, J. (2012). Temporal networks. Physics Reports, 519, 97-125.

Jordan, J., Yoeli, E., \& Rand, D. (2020). Don't get it or don't spread it? Comparing self-interested versus prosocially framed COVID-19 prevention messaging. https://psyarxiv.com/yuq7x

Jordan, J. J., \& Rand, D. G. (2020). Signaling when no one is watching: A reputation heuristics account of outrage and punishment in one-shot anonymous interactions. Journal of Personality and Social Psychology, 118, 57-88.

Jørgensen, F. J., Bor, A., \& Petersen, M. B. (2020). Compliance without fear: Predictors of protective behavior during the first wave of the COVID-19 pandemic. British Journal of Health Psychology, 26, 679-696.

Jung, H., \& Albarracín, D. (2021). Concerns for others increases the likelihood of vaccination against influenza and COVID-19 more in sparsely rather than densely populated areas. Proceedings of the National Academy of Sciences, 118, e2007538118. 
Kaplan, R. M., \& Milstein, A. (2021). Influence of a COVID-19 vaccine's effectiveness and safety profile on vaccination acceptance. Proceedings of the National Academy of Sciences, 118(10), e2021726118.

Kimbrough, E. O., \& Vostroknutov, A. (2016). Norms make preferences social. Journal of the European Economic Association, 14, 608-638.

Korn, L., Böhm, R., Meier, N. W., \& Betsch, C. (2020). Vaccination as a social contract. Proceedings of the National Academy of Sciences, 117, 14890-14899.

Krupp, D. B., Debruine, L. M., \& Barclay, P. (2008). A cue of kinship promotes cooperation for the public good. Evolution and Human Behavior, 29, 49-55.

Kvarven, A., Strømland, E., Wollbrant, C., Andersson, D., Johannesson, M., Tinghög, G., ... \& Myrseth, K. O. R. (2020). The intuitive cooperation hypothesis revisited: a meta-analytic examination of effect size and between-study heterogeneity. Journal of the Economic Science Association, 6, 26-42.

Latora, V., Nicosia, V., \& Russo, G. (2017). Complex networks: principles, methods and applications. Cambridge University Press.

Levine, E. E., Barasch, A., Rand, D., Berman, J. Z., \& Small, D. A. (2018). Signaling emotion and reason in cooperation. Journal of Experimental Psychology: General, 147, 702-719.

Loomba, S., de Figueiredo, A., Piatek, S. J., de Graaf, K., \& Larson, H. J. (2021). Measuring the impact of COVID-19 vaccine misinformation on vaccination intent in the UK and USA. Nature Human Behaviour, 5, 337-348.

Lu, J. G., Jin, P., \& English, A. S. (2021). Collectivism predicts mask use during COVID-19. Proceedings of the National Academy of Sciences, 118.

Lunn, P. D., Timmons, S., Barjaková, M., Belton, C. A., Julienne, H., \& Lavin, C. (2020). Motivating social distancing during the Covid-19 pandemic: An online experiment. Social Science \& Medicine, 113478.

Milinski, M., Semmann, D., Bakker, T. C., \& Krambeck, H. J. (2001). Cooperation through indirect reciprocity: image scoring or standing strategy? Proceedings of the Royal Society: Biological Sciences, 268, 2495-2501.

Mitze, T., Kosfeld, R., Rode, J., \& Wälde, K. (2020). Face masks considerably reduce COVID-19 cases in Germany. Proceedings of the National Academy of Sciences, 117, 32293-32301.

Morgenstern, O., \& Von Neumann, J. (1953). Theory of games and economic behavior. Princeton university press.

Moxnes, E., \& Van der Heijden, E. (2003). The effect of leadership in a public bad experiment. Journal of Conflict Resolution, 47, 773-795.

Nax, H. H., Perc, M., Szolnoki, A., \& Helbing, D. (2015). Stability of cooperation under image scoring in group interactions. Scientific Reports, 5, 12145.

Nivette, A., Ribeaud, D., Murray, A., Steinhoff, A., Bechtiger, L., Hepp, U., ... \& Eisner, M. (2021). Non-compliance with COVID-19-related public health measures among young adults in Switzerland: Insights from a longitudinal cohort study. Social Science $\&$ Medicine, 268, 113370.

Nowak, M. A. (2006). Five rules for the evolution of cooperation. Science, 314, 1560-1563. 
Nowak, M. A., \& May, R. M. (1992). Evolutionary games and spatial chaos. Nature, 359, 826-829.

Nowak, M. A., \& Sigmund, K. (1998). Evolution of indirect reciprocity by image scoring. Nature, 393, 573-577.

Nowak, M. A., \& Sigmund, K. (2005). Evolution of indirect reciprocity. Nature, 437, 1291-1298.

Perc, M., Gómez-Gardenes, J., Szolnoki, A., Floría, L. M., \& Moreno, Y. (2013). Evolutionary dynamics of group interactions on structured populations: a review. Journal of the Royal Society Interface, 10, 20120997.

Perc, M., Jordan, J. J., Rand, D. G., Wang, Z., Boccaletti, S., \& Szolnoki, A. (2017). Statistical physics of human cooperation. Physics Reports, 687, 1-51.

Perc, M., \& Szolnoki, A. (2010). Coevolutionary games - a mini review. BioSystems, 99, $109-125$

Perc, M., \& Szolnoki, A. (2008). Social diversity and promotion of cooperation in the spatial prisoner's dilemma game. Physical Review E, 77, 011904.

Pfattheicher, S., Nockur, L., Böhm, R., Sassenrath, C., \& Petersen, M. B. (2020). The emotional path to action: Empathy promotes physical distancing during the COVID-19 pandemic. Psychological Science, 31, 1363-1373.

Pfattheicher, S., Petersen, M. B., \& Böhm, R. (in press). Information about herd immunity through vaccination and empathy promote COVID-19 vaccination intentions. Health Psychology. Preprint: https://doi.org/10.31234/osf.io/wzu6k

Rand, D. G. (2016). Cooperation, fast and slow: Meta-analytic evidence for a theory of social heuristics and self-interested deliberation. Psychological Science, 27, 1192-1206.

Rand, D. G., \& Kraft-Todd, G. T. (2014). Reflection does not undermine self-interested prosociality. Frontiers in Behavioral Neuroscience, 8, 300.

Rand, D. G., Peysakhovich, A., Kraft-Todd, G. T., Newman, G. E., Wurzbacher, O., Nowak, M. A., \& Greene, J. D. (2014). Social heuristics shape intuitive cooperation. Nature communications, 5, 3677.

Santos, F. C., \& Pacheco, J. M. (2005). Scale-free networks provide a unifying framework for the emergence of cooperation. Physical Review Letters, 95, 098104.

Santos, F. C., Pacheco, J. M., \& Lenaerts, T. (2006). Cooperation prevails when individuals adjust their social ties. PLoS Computational Biology, 2, 1284-1290.

Santos, F. C., Pinheiro, F. L., Lenaerts, T., \& Pacheco, J. M. (2012). The role of diversity in the evolution of cooperation. Journal of Theoretical Biology, 299, 88-96

Santos, F. C., Santos, M. D., \& Pacheco, J. M. (2008). Social diversity promotes the emergence of cooperation in public goods games. Nature, 454, 213-216.

Schwarzinger, M., Watson, V., Arwidson, P., Alla, F., \& Luchini, S. (2021). COVID-19 vaccine hesitancy in a representative working-age population in France: a survey experiment based on vaccine characteristics. The Lancet Public Health, 6(4), e210-e221.

Sjåstad, H. (2019). Short-sighted greed? Focusing on the future promotes reputation-based generosity. Judgment and Decision Making, 14, 199-213. 
Sprengholz, P.; Betsch, C., \& Böhm, R. (in press). Reactance revisited: Consequences of mandatory and scarce vaccination in the case of COVID-19. Applied Psychology: Health \& Well-Being.

Szolnoki, A., Perc, M., \& Szabó, G. (2009). Topology-independent impact of noise on cooperation in spatial public goods games. Physical Review E, 80, 056109.

Tarnita, C. E., Antal, T., Ohtsuki, H., \& Nowak, M. A. (2009). Evolutionary dynamics in set structured populations. Proceedings of the National Academy of Sciences of the USA, 106, 8601-8604.

Trivers, R. L. (1971). The evolution of reciprocal altruism. The Quarterly Review of Biology, 46, 35-57.

Van Bavel, J. J., Baicker, K., Boggio, P. S., Capraro, V., Cichocka, A., Cikara, M., ... \& Willer, R. (2020a). Using social and behavioural science to support COVID-19 pandemic response. Nature Human Behaviour, 4, 460-471.

Van Bavel, J. J., et al. (2020b). National identity predicts public health support during a global pandemic: Results from 67 nations. https://doi.org/10.31234/osf.io/ydt9

Van Der Linden, C., \& Savoie, J. (2020). Does collective interest or self-interest motivate mask usage as a preventive measure against COVID-19? Canadian Journal of Political Science, 53, 391-397.

Van Lange, P. A., Klapwijk, A., \& Van Munster, L. M. (2011). How the shadow of the future might promote cooperation. Group Processes \& Intergroup Relations, 14, 857-870.

Vinck, P., Pham, P. N., Bindu, K. K., Bedford, J., \& Nilles, E. J. (2019). Institutional trust and misinformation in the response to the 2018-19 Ebola outbreak in North Kivu, DR Congo: a population-based survey. The Lancet Infectious Diseases, 19(5), 529-536.

Vonasch, A. J., \& Sjåstad, H. (2021). Future-orientation (as trait and state) promotes reputation-protective choice in moral dilemmas. Social Psychological and Personality Science, 12, 383-391.

Wang, Z., Bauch, C. T., Bhattacharyya, S., d'Onofrio, A., Manfredi, P., Perc, M., ... \& Zhao, D. (2016). Statistical physics of vaccination. Physics Reports, 664, 1-113.

Wang, Z., Szolnoki, A., \& Perc, M. (2012). Evolution of public cooperation on interdependent networks: The impact of biased utility functions. Europhysics Letters, 97, 48001.

Wells, C. R., Huppert, A., Fitzpatrick, M. C., Pandey, A., Velan, B., Singer, B. H., ... \& Galvani, A. P. (2020). Prosocial polio vaccination in Israel. Proceedings of the National Academy of Sciences, 117, 13138-13144.

Zaki, J. (2020). Catastrophe compassion: Understanding and extending prosociality under crisis. Trends in Cognitive Sciences, 24, 587-589. 
